\title{
TRAP1 regulates cell cycle and apoptosis in thyroid carcinoma cells
}

\author{
Giuseppe Palladino,**, Tiziana Notarangelo2,*, Giuseppe Pannone ${ }^{3}$, \\ Annamaria Piscazzi', Olga Lamacchia4, Lorenza Sisinni², Girolamo Spagnoletti', \\ Paolo Toti5, Angela Santoro6, Giovanni Storto7, Pantaleo Bufo3, Mauro Cignarelli4, \\ Franca Esposito ${ }^{8}$ and Matteo Landriscina1,2
}

'Medical Oncology Unit, Department of Medical and Surgical Sciences, University of Foggia, Foggia, Italy 2Laboratory of Pre-Clinical and Translational Research, IRCCS, Referral Cancer Center of Basilicata, Rionero in Vulture, Potenza, Italy

${ }^{3}$ Anatomic Pathology Unit, Department of Clinic and Experimental Medicine, University of Foggia, Foggia, Italy ${ }^{4}$ Endocrinology Unit, Department of Medical and Surgical Sciences, University of Foggia, Foggia, Italy 5Pathology Unit, Department of Human Pathology and Oncology, University of Siena, Siena, Italy GInstitute of Histopathology and Diagnostic Cytopathology, Fondazione di Ricerca e Cura 'Giovanni Paolo II' UCSC, Campobasso, Italy

${ }^{7}$ Nuclear Medicine Unit, IRCCS, Referral Cancer Center of Basilicata, Rionero in Vulture, Potenza, Italy ${ }^{8}$ Department of Molecular Medicine and Medical Biotechnology, University of Naples Federico II, Naples, Italy *(G Palladino and T Notarangelo contributed equally to this work)

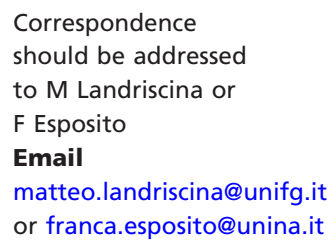

\begin{abstract}
Tumor necrosis factor receptor-associated protein 1 (TRAP1) is a heat shock protein 90 (HSP90) molecular chaperone upregulated in several human malignancies and involved in protection from apoptosis and drug resistance, cell cycle progression, cell metabolism and quality control of specific client proteins. TRAP1 role in thyroid carcinoma (TC), still unaddressed at present, was investigated by analyzing its expression in a cohort of 86 human TCs and evaluating its involvement in cancer cell survival and proliferation in vitro. Indeed, TRAP1 levels progressively increased from normal peritumoral thyroid gland, to papillary TCs (PTCs), follicular variants of PTCs (FV-PTCs) and poorly differentiated TCs (PDTCs). By contrast, anaplastic thyroid tumors exhibited a dual pattern, the majority being characterized by high TRAP1 levels, while a small subgroup completely negative. Consistently with a potential involvement of TRAP1 in thyroid carcinogenesis, TRAP1 silencing resulted in increased sensitivity to paclitaxel-induced apoptosis, inhibition of cell cycle progression and attenuation of ERK signaling. Noteworthy, the inhibition of TRAP1 ATPase activity by pharmacological agents resulted in attenuation of cell proliferation, inhibition of ERK signaling and reversion of drug resistance. These data suggest that TRAP1 inhibition may be regarded as potential strategy to target specific features of human TCs, i.e., cell proliferation and resistance to apoptosis.
\end{abstract}

$\begin{aligned} & \text { Key Words } \\ & \text { TRAP1 } \\ - & \text { thyroid carcinoma } \\ - & \text { apoptosis } \\ - & \text { cell cycle } \\ & \text { HSP990 }\end{aligned}$

Endocrine-Related Cancer (2016) 23, 699-709

\section{Introduction}

The vast majority of thyroid carcinomas (TCs) are well-differentiated tumors, usually curable by the combination of surgery, radioiodine ablation and long-term thyroid-stimulating hormone (TSH) suppressive therapy (Mazzaferri \& Massoll 2002). A small subset of thyroid tumors are de novo poorly 
differentiated carcinomas or arises from the (de) differentiation of initially well-differentiated carcinomas and are characterized by a more aggressive behavior, earlier metastatic spread, and loss of TSH signaling, cell differentiation and iodine retention capacity (Regalbuto et al. 2012).

The efficacy of radioiodine therapy relies on the capacity of TC cells to uptake and retain intracellular radioiodine and this is exclusively dependent on the expression of the sodium-iodide symporter (NIS) (Kogai \& Brent 2012). NIS is a basolateral membrane protein primarily regulated at transcriptional level by TSH, through the TSH receptor (TSHR) signaling, and by TSHindependent mechanisms (Kogai \& Brent 2012, Mu et al. 2012). In such a context, radioiodine-refractory thyroid tumors are poorly differentiated carcinomas, characterized by poor or absent NIS expression, unresponsiveness to radioiodine therapy and other cytotoxics, increased metastatic spread and, thus, worst prognosis and limited therapeutic options (Regalbuto et al. 2012). Indeed, the activation of RAF/ERK signaling pathway is a major mechanism responsible for progression toward radioiodine-refractory and apoptosis-resistant phenotypes (Nikiforov et al. 2011). Therefore, several attempts have been made to study the molecular mechanisms responsible for the more aggressive phenotype of radioiodinerefractory thyroid tumors with the aim to find molecular targets for novel, specific and more effective therapies (Haugen 2004, Alonso-Gordoa et al. 2015).

Tumor necrosis factor receptor-associated protein 1 (TRAP1) is a member of the heat shock protein 90 (HSP90) family of molecular chaperones, involved in prosurvival signaling of cancer cells, protection of mitochondria against oxidative stress, drug resistance, cell cycle regulation, metabolic reprogramming and regulation of protein homeostasis through the translational quality control of specific client proteins (Amoroso et al. 2014). Noteworthy, recent evidence suggests that TRAP1 modulates ERK signaling, likely through the regulation of BRAF synthesis/ubiquitination (Condelli et al. 2014). TRAP1 is upregulated in various tumor types, including colorectal, breast, lung, and prostate cancers (Costantino et al. 2009, Leav et al. 2010, Maddalena et al. 2013, Agorreta et al. 2014) and in some of these malignancies, such as prostate or colorectal carcinomas, TRAP1 levels correlate with poor clinical outcome, higher grading and/ or advanced stages (Leav et al. 2010, Han et al. 2014), thus supporting a role of TRAP1 in cancer promotion and progression (Amoroso et al. 2014, Rasola et al. 2014). Conversely, other studies questioned the oncogenic role of TRAP1, suggesting that TRAP1 levels correlate inversely with malignant progression and tumor grading in specific human malignancies, i.e., ovary, cervical, bladder and clear cell renal cancers (Aust et al. 2012, Yoshida et al. 2013, Rasola et al. 2014, Matassa et al. 2016). Herein, the expression of TRAP1 was evaluated for the first time in a series of human thyroid tumors ranging from well-differentiated to poorly differentiated and anaplastic carcinomas. In addition, the effect of TRAP1 knockdown on cell cycle control and protection from apoptosis in vitro was evaluated in this tumor type.

\section{Patients and methods}

\section{Study population}

Eighty-six patients who underwent total thyroidectomy at the Universities of Foggia or Siena (Italy) were selected for this study. Thirty-eight of them were affected by papillary TC (PTC), 20 by follicular variant of PTC (FV-PTC), 12 by poorly differentiated TC (PDTC) and 16 by anaplastic TC (ATC). All of them received surgical treatment with curative intent between 2002 and 2014. Patient's characteristics, including clinical data referring to sex, age, stage, degree of differentiation, and uni/multifocality are reported in Table 1 , as mean \pm s.D. for continuous variables and numbers and percentages for categorical ones. All patients gave their written informed consent to use biological specimens for investigational procedures after full explanation of the purpose and nature of the study. Demographical and clinical data were extracted from clinical records. The histopathological diagnosis was carefully reviewed at the Department of Clinical and Experimental Medicine, Section of Anatomic Pathology of the University of Foggia. Tumor extent was revised and classified according to the classification system of the American Joint Committee on Cancer (AJCC) (Wada et al. 2007).

\section{Immunohistochemistry}

$4-\mu \mathrm{m}$ serial sections from formalin-fixed paraffinembedded blocks were cut and mounted on polyL-lysine-coated glass slides. Immunohistochemical analysis was performed using a Benchmark autostainer (Ventana Medical Systems, Tucson, AZ, USA) and/or manual standard linked streptavidin-biotin horseradish peroxidase technique (LSAB-HRP), according to the best protocol for the antibody previously tested in our laboratory (Pannone et al. 2014). Sections were

Published by Bioscientifica Ltd. 
Table 1 Demographic and clinicopathological characteristics of patients.

\begin{tabular}{l}
\hline \\
\hline Cases (\%) \\
Sex \\
Male \\
Female \\
Age (mean \pm S.D.) \\
TNM stage (\%) \\
I \\
II \\
III \\
IV \\
Tumor size - cm (mean \pm s.D.) \\
Tumors with invasive growth (\%) \\
Multifocal tumors (\%) \\
Tumors with lymph node metastases (\%) \\
Tumors with distant metastases (\%) \\
Tumors with positive resection margins (\%) \\
Relapses (\%)
\end{tabular}

\begin{tabular}{c} 
PTC \\
\hline $38(44.2)$ \\
14 \\
24 \\
$50.7 \pm 14.5$ \\
$24(63.1)$ \\
$2(5.2)$ \\
$8(21.0)$ \\
$4(10.5)$ \\
$1.34 \pm 1.1$ \\
$24(63.1)$ \\
$7(18.4)$ \\
$9(23.6)$ \\
$2(5.2)$ \\
$10(26.3)$ \\
$6(15.7)$ \\
\end{tabular}

\begin{tabular}{c}
\hline FV-PTC \\
\hline $20(23.2)$ \\
\\
8 \\
12 \\
$48.7 \pm 16.2$ \\
$12(60.0)$ \\
0 \\
$5(25.0)$ \\
$3(15.0)$ \\
$1.72 \pm 1.2$ \\
$11(55.0)$ \\
$6(30.0)$ \\
$8(40.0)$ \\
$1(5.0)$ \\
$7(35.0)$ \\
$4(20.0)$ \\
\hline
\end{tabular}

$\frac{\text { PDTC }}{12(14.0)}$

$\frac{\text { ATC }}{16(18.6)} \stackrel{P \text { value }}{ }$

FV-PTC, follicular variant of papillary thyroid carcinoma; PDTC, poorly differentiated thyroid carcinoma; PTC, papillary thyroid carcinoma.

incubated with mouse monoclonal anti-TRAP1 antibody (1:750 dilution) (sc-73604; Santa Cruz Biotechnology). Negative controls were performed omitting primary antibody. Sections were counterstained with Gill's type 2 hematoxylin, dehydrated with ethanol and permanently coverslipped. Results of the immunohistochemical staining were evaluated separately by two observers (P B and G P), particularly trained for thyroid pathology and immunohistochemistry and completely blind to the histological diagnosis. The inter-rater reliability between the two investigators examining the immunostained sections was assessed by the Cohen's $K$ test, yielding $K$ values $>0.70$ in all instances. Normal distribution of the data was analyzed by the Kolmogorov-Smirnov test. Immune-stained cells were counted in at least $10 \mathrm{High}$ Power Fields (HPF) analyzed with an optical microscope (Olympus BX53; Olympus Italia, Milan, Italy) at 40x magnification. TRAP1-positive staining was defined as cytoplasmic staining in normal peritumoral noninfiltrated and tumor tissue. Peritumoral noninfiltrated thyroid gland was classified in microfollicular and macrofollicular areas based on the prevalence of, respectively, small follicles with sparse colloid or large follicles with dense colloid (Motta 1984). The number of TRAP1-expressing tumor cells was estimated as a mean percentage of total number of cells per section and grouped according to the percentage of positive cells: 0 (no staining), 1 (1-25\%), 2 (26-50\%), 3 (51-75\%), and 4 (76-100\%). The intensity of TRAP1 staining was graded as 0 (no staining), 1 (weak), 2 (moderate), or 3 (strong). A combined numeric IHC score was calculated as the product of staining intensity and percentage of stained cells and reported as mean and S.D. (Smith et al. 2014).

\section{Cell cultures, constructs, siRNAs and chemicals}

Follicular ML1 and FTC133, papillary BCPAP, and anaplastic BHT101 and CAL62 TC cells below passage 20 were used for this study. BCPAP and CAL62 cells were cultured in DMEM supplemented with $10 \%$ (vol/vol) fetal bovine serum (FBS), $1.5 \mathrm{mM}$ glutamine, and $100 \mathrm{U} / \mathrm{mL}$ penicillin and streptomycin, ML1 cells in the same medium supplemented with $1 \mathrm{mM}$ sodium pyruvate, BHT101 cells in the same medium supplemented with $20 \%$ (vol/ vol) FBS. FTC133 cells were cultured in DMEM:Ham's F12 (1:1) supplemented with 10\% (vol/vol) FBS, $1.5 \mathrm{mM}$ glutamine, and $100 \mathrm{U} / \mathrm{mL}$ penicillin and streptomycin. All media were supplemented with TSH $(10 \mathrm{U} / \mathrm{mL})$. All cell lines cells were purchased from Leibniz Institute DSMZ-German Collection of Microorganisms and Cell Culture (DSMZ, Braunschweig, Germany) with the exception of FTC133 cells that were purchased from Sigma-Aldrich. Cell lines were routinely monitored in our laboratory by microscopic morphology, cell line authentication was verified by evaluating BRAF and KRAS mutational status by pyrosequencing. Full-length TRAP1 construct was obtained as described previously (Landriscina et al. 2010). Transient transfection of DNA plasmid was performed with PolyFect Transfection Reagent (Qiagen), according to the manufacturer's protocol. siRNAs of TRAP1 and TBP7 were purchased from Qiagen (Cat. No. SI00115150 for TRAP1,

Published by Bioscientifica Ltd 
Cat. No. SI00301469 for TBP7). For control experiments, cells were transfected with a similar amount of control siRNA (Qiagen, Cat. No. SI03650318). For knockdown experiments, siRNAs were diluted to a final concentration of $40 \mathrm{nM}$ and transiently transfected by the HiPerFect Transfection Reagent (Qiagen), according to manufacturer's protocol. Unless otherwise specified, reagents were purchased from Sigma-Aldrich, with HSP990 kindly provided by Novartis Oncology (Origgio, VA, Italy) and gamitrinib by Dr Altieri (The Wistar Institute, Philadelphia, PA, USA).

\section{Immunoblot analysis}

Total cell lysates were obtained by homogenization of cell pellets and tissue samples in cold lysis buffer (20 mmol/L Tris (pH 7.5) containing $300 \mathrm{mmol} / \mathrm{L}$ sucrose, $60 \mathrm{mmol} / \mathrm{L} \mathrm{KC1,} 15 \mathrm{mmol} / \mathrm{L} \mathrm{NaC1}$, 5\% (vol/vol) glycerol, $2 \mathrm{mmol} / \mathrm{L}$ EDTA, 1\% (vol/vol) Triton X100, $1 \mathrm{mmol} / \mathrm{L}$ phenylmethylsulfonylfluoride, $2 \mathrm{mg} / \mathrm{mL}$ aprotinin, $2 \mathrm{mg} / \mathrm{mL}$ leupeptin, and $0.2 \%$ (wt/vol) deoxycholate) for $1 \mathrm{~min}$ at $4^{\circ} \mathrm{C}$ and further sonication for an additional $30 \mathrm{~s}$ at $4^{\circ} \mathrm{C}$. Protein concentration was quantified using the Bio-Rad Protein Assay Kit (Bio-Rad Laboratories), according to the manufacturer's procedures. Samples were resolved on SDS-PAGE, transferred on nitrocellulose membrane (Bio-Rad Laboratories), and immunoblotted with the following mouse monoclonal antibodies from Santa Cruz Biotechnology: anti-TRAP1 (sc-73604), anti-BRAF (sc5284), anti-TBP7 (sc-166003) and antiglyceraldehyde3-phosphate dehydrogenase (GAPDH, sc-69778). The following antibodies were also used: mouse monoclonal antiphospho 44/42 MAPK (pERK1/2, \#9106), rabbit monoclonal anti-eIF4E (\#C46H6) from Cell Signaling Technology, rabbit polyclonal anti-MAPK ERK1/2 from Calbiochem (\#442704). Rabbit polyclonal anti-Sorcin antibody was kindly provided by Prof E Chiancone (University of Rome 'La Sapienza'). Specific bands were revealed using the Clarity Western ECL Substrate (Bio-Rad Laboratories).

\section{Cell cycle analysis}

Cells were incubated in a culture medium supplemented with $20 \mathrm{mmol} / \mathrm{L}$ 5-bromo-20-deoxyuridine (BrdUrd) for $20 \mathrm{~min}$ and harvested. Subsequent to incubation in a solution containing $3 \mathrm{~N} \mathrm{HCl}$ for $30 \mathrm{~min}$ at room temperature to obtain DNA denaturation, cell pellets were further incubated in the presence of anti-BrdUrd FITC-conjugated antibody (Becton Dickinson, Milan,
Italy) for $1 \mathrm{~h}$ at room temperature in the darkness. After washing with PBS, cells were further incubated with $6 \mathrm{mg} / \mathrm{mL}$ propidium iodate (PI) for $20 \mathrm{~min}$ and then evaluated using FACSCalibur (Becton Dickinson).

\section{Apoptosis assay}

Apoptosis was evaluated by cytofluorimetric analysis of Annexin- $\mathrm{V}$ and 7-amino-actinomycin-D (7-AAD)-positive cells using the FITC-Annexin-V/7-AAD kit (Beckman Coulter, Milan, Italy). Stained cells were analyzed using the FACSCalibur (Becton Dickinson). Positive staining for Annexin-V as well as double staining for Annexin- $V$ and 7-AAD was interpreted as signs of early and late phases of apoptosis, respectively (Maddalena et al. 2011).

\section{Statistical analysis}

The paired Student's t-test was used to establish the statistical significance in apoptosis and cell cycle distribution between silenced and control cells or drugand vehicle-treated cells. Statistically significant values $(P<0.05)$ are reported in the Figure legends. All experiments were independently performed at least three times.

Mean differences in IHC score and other variables were compared by unpaired Student's $t$-test or one-way ANOVA F-tests, as appropriate. Differences between categorical variables were tested by Pearson's $\chi^{2}$. The statistical packaged SPSS version 13.0 (SPSS) was used. A $P$ value $<0.05$ was considered to be significant.

\section{Results}

\section{TRAP1 expression is progressively upregulated from normal thyroid to PDTC and FV-PTCs}

TRAP1 expression was evaluated in a series of 86 thyroid tumors ranging from well-differentiated to poorly differentiated and anaplastic TCs, subdivided in 38 PTCs, 20 FV-PTCs, 12 PDTCs, and 16 ATCs. TRAP1 expression was visualized as cytoplasmic staining in normal peritumoral noninfiltrated thyroid gland and tumor cells and expressed as IHC score. Representative IHC images are reported in Fig. 1A, whereas the results of immunohistochemical staining according to histological subtypes are shown in Fig. $1 \mathrm{~B}$ and $\mathrm{C}$. TRAP1 expression was also confirmed by immunoblot analysis in selected tumors (Supplementary Fig. 1A, see section on supplementary data given at the end of this article). TRAP1 was detectable, although at low level,

Published by Bioscientifica Ltd 
A
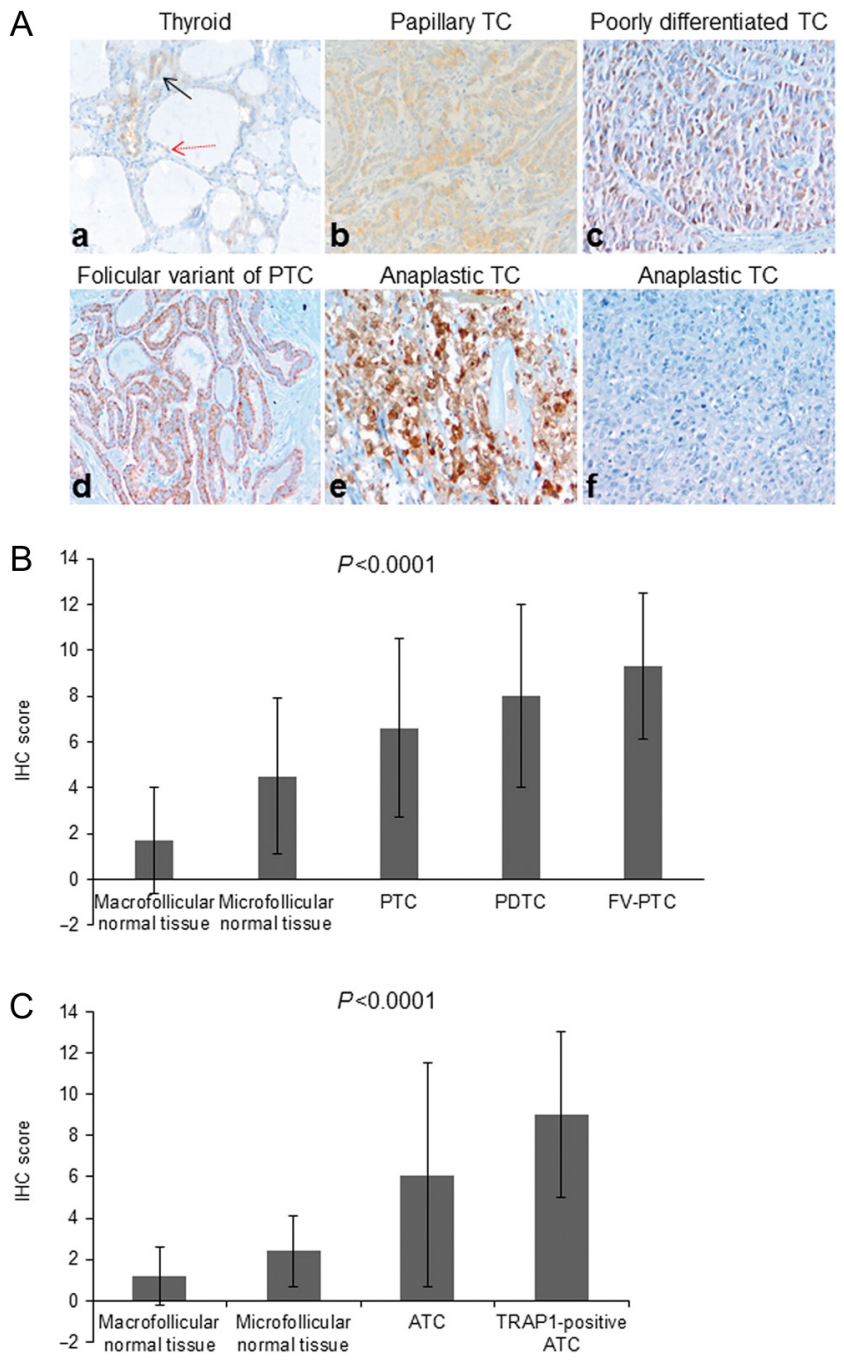

Figure 1

TRAP1 expression is progressively upregulated from normal thyroid gland to PTCs, PDTCs and FV-PTCs. (A) TRAP1 IHC staining in representative cases of noninfiltrated macrofollicular (red arrow) and microfollicular (black arrow) areas of thyroid gland (a), papillary thyroid carcinoma (b), poorly differentiated thyroid carcinoma (c), follicular variant of papillary thyroid carcinoma (d) and anaplastic thyroid carcinoma (e and f); automated LSAB-HRP technique, counterstaining with Gill's type 2 hematoxylin, original magnifications 100x. (B and C) TRAP1 IHC scores in microfollicular and macrofollicular noninfiltrated normal thyroid tissue, PTCs, PDTCs and FV-PTCs (B) and in ATCs compared with macrofollicular and microfollicular normal thyroid gland (C).

in noninfiltrated peritumoral normal thyroid gland, being its expression higher in microfollicular structures compared with macrofollicular areas $(P<0.0001$; Fig. $1 \mathrm{~A}$, panel a and Fig. 1B). TRAP1 was also detectable in all PTCs, PDTCs, and FV-PTCs, with a statistically significant progressive increase in TRAP1 IHC score from normal thyroid to PTCs, PDTCs, and FV-PTCs $(P<0.0001$; Fig. 1A, panels b-d and Fig. 1B). This difference between PTCs, PDTCs, and FV-PTCs is statistically significant also after excluding normal thyroid gland from the analysis $(P=0.04$; Supplementary Fig. 1B). The IHC tumor tissue score of TRAP1 was also significantly higher in PTCs, FV-PTCs, and PDTCs, each compared independently with respective macrofollicular and microfollicular peritumoral thyroid tissues $(P<0.0001$; Supplementary Fig. 1C, D and E), whereas only FV-PTCs showed significantly higher TRAP1 levels compared with PTCs $(P=0.012)$. Among 16 ATCs, TRAP1 was highly expressed in 11 out of 16 cases, 5 ATCs being completely negative upon TRAP1 immunostaining (Fig. 1A, compare panels e and f). For this peculiar and unexpected pattern, ATCs were analyzed independently of other subgroups: indeed, TRAP1 IHC tissue score was significantly higher in the whole cohort of ATCs and in the 11 TRAP1-positive ATCs compared with respective macrofollicular and microfollicular peritumoral areas $(P<0.0001$; Fig. $1 C)$. Remarkably, 5 out of 16 ATCs showed lack of TRAP1 expression, below normal thyroid gland levels (Fig. 1, panel f). These data suggest that TRAP1 expression is increased in TCs compared with normal thyroid and that there is trend for a progressive increase of TRAP1 levels from PTCs to PDTCs and FV-PTCs.

\section{TRAP1 regulates survival in follicular and anaplastic TC cells}

FV-PTCs are characterized by a more aggressive behavior compared with classical PTCs (Vivero et al. 2013, Yu et al. 2013), as well as PDTCs are aggressive tumors with poor prognosis and lack of response to radioiodine (Patel \& Shaha 2014). Consistently, a progressive and statistically significant increase of tumor size and tumor stage was observed from PTCs to FV-PTCs, PDTCs and ATCs $(P<0.0001$ for both variables; Table 1$)$ in our series of 86 TCs. Thus, to evaluate the role of TRAP1 pathway in TC progression, the correlation between TRAP1 expression and specific features of more aggressive/less differentiated TC cells was analyzed in a panel of TC cell lines: i.e., follicular ML1 and FTC133, papillary BCPAP, and anaplastic BHT101 and CAL62 carcinoma cells. Indeed, BHT101 and BCPAP cells are characterized by the BRAFV600E mutation, CAL62 cells by the KRAS G12R mutation (Piscazzi et al. 2012), whereas ML1 and FTC133 cells by a wild-type genotype for both genes. Based on the well-established role of TRAP1 in inducing cytoprotective responses by inhibiting mitochondrial apoptotic pathway (Kang et al. 2007, Montesano Gesualdi et al. 2007), TRAP1 was silenced in our panel of TC cell lines to assess protection from paclitaxel-induced apoptosis (Fig. 2A).

Published by Bioscientifica Ltd 
A significant increase of apoptotic cell death was observed in TRAP1-silenced cell lines upon exposure to paclitaxel (Fig. 2B). In concomitant experiments, apoptotic cell death was assessed upon exposure of TC cell lines to gamitrinib, a dual HSP90/TRAP1 inhibitor engineered to accumulate into mitochondria and counteract TRAP1dependent inhibition of mitochondrial transition pore (Kang et al. 2009). Indeed, gamitrinib induced apoptosis in a dose-dependent manner (Fig. 2C) and, consistently, the pretreatment with subcytotoxic doses of gamitrinib significantly enhanced paclitaxel-induced apoptosis in TC cell lines (Fig. 2D). Different from what was observed in colon carcinoma cells (Condelli et al. 2015), gamitrinib cytotoxicity was independent of BRAF mutational status in TC cells. These data suggest that TRAP1 is responsible for enhancing the apoptotic threshold of TC cells, thus, inducing resistance to chemotherapeutics.

\section{TRAP1 regulates cell cycle progression and ERK signaling in TC cells lines}

Previous evidences suggest an involvement of TRAP1 in cell cycle regulation in colon and breast carcinoma cells (Condelli et al. 2014). Therefore, the role of TRAP1 in favoring cell cycle progression was assessed in TC cell lines. Indeed, TRAP1 silencing resulted in a modest, but significant attenuation of cell proliferation (Fig. 3A) and this correlated with a parallel significant inhibition of S-phase, with accumulation of cells in G0-G1 and/or G2-M phase, depending on the specific TC cell line (Fig. 3B).

Previous evidences by our group suggest that TRAP1 is responsible for BRAF quality control and regulation of downstream ERK signaling (Condelli et al. 2014), a key event in TC progression (Nikiforov et al. 2011). Therefore, BRAF expression levels and ERK phosphorylation were evaluated upon TRAP1 silencing in TC cells. Indeed, TRAP1 interference induced a strong downregulation of ERK signaling in all TC cell lines and this was paralleled by the expected downregulation of BRAF protein expression in ML1, FTC133, BHT101 and BCPAP cells (Fig. 4A). Surprisingly, ERK phosphorylation was significantly downregulated, independently from BRAF expression, in KRAS-mutated CAL62 TC cells (Fig. 4A). These data suggest that TRAP1 control of cell cycle progression correlates with regulation of ERK signaling.

\section{TRAP1 targeting results in inhibition of ERK signaling and cell proliferation in TC cell lines}

We recently demonstrated that the dual HSP90/ TRAP1 inhibitor HSP990, that in previous studies was shown to inhibit TRAP1 ATPase domain (Menezes et al. 2012), antagonizes TRAP1 chaperoning activity toward its client proteins and acts as cytostatic agent in BRAF-mutated colon carcinoma cells (Condelli et al. 2014). Hence, this agent was used to further validate TRAP1 as a potential therapeutic target in TC cells. In preliminary experiments, HSP990 was confirmed to induce the downregulation of TRAP1-specific client proteins (i.e., $18 \mathrm{kDa}$ Sorcin and eIF4E) (Landriscina et al. 2010, Matassa et al. 2013) in ML1, CAL62 (Fig. 4B), and BHT101 (Supplementary Fig. 2A) TC cells, thus resembling the phenotype obtained by TRAP1 and TBP7 dual silencing (Fig. 4C). Consistently, HSP990 induced a parallel attenuation of BRAF protein expression and ERK phosphorylation (Fig. 4D), significantly inhibiting
A
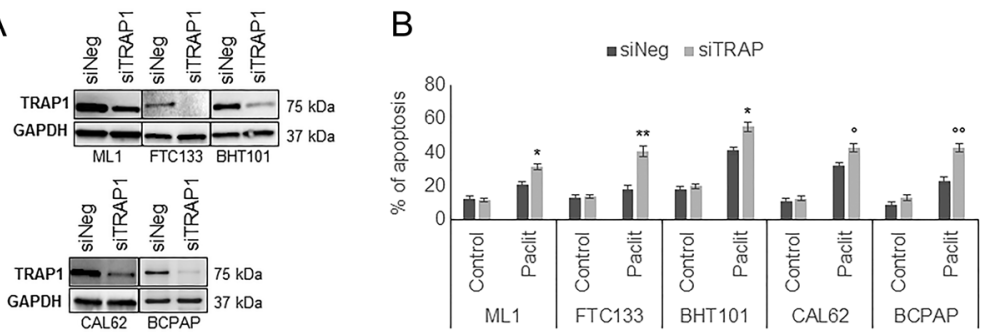

C घControl $₫ 1 \mu \mathrm{M}$ Gamitrinib $\quad ₫ 10 \mu \mathrm{M}$ Gamitrinib

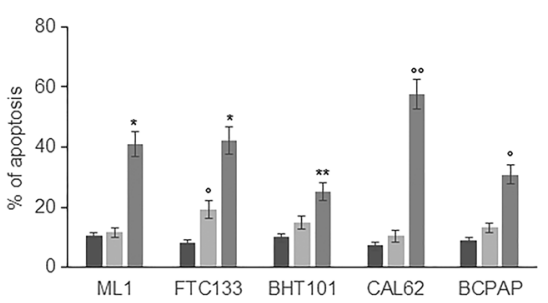

D

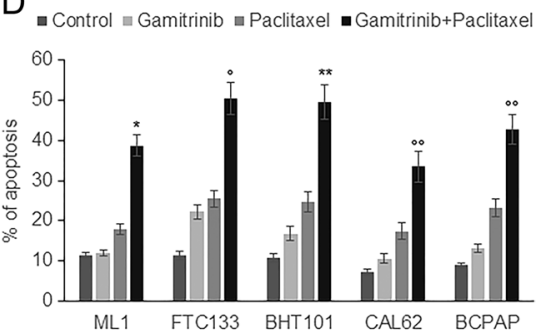

Figure 2

TRAP1 protects from apoptosis. (A and B) TRAP1 expression (A) and apoptotic levels (B) in $\mathrm{ML1}$, FTC133, BHT101, CAL62 and BCPAP cells transfected with control and TRAP1 siRNAs and exposed to $1 \mu \mathrm{M}$ paclitaxel for $24 \mathrm{~h}$. Statistical significance with respect to negative siRNA cells treated with paclitaxel: ${ }^{*} P=0.002 ; * * P=0.0003$; ${ }^{\circ} P=0.005 ;{ }^{\circ} P=0.004$. (C) Apoptotic levels in ML1, FTC133, BHT101, CAL62, and BCPAP cells treated with 1 and $10 \mu \mathrm{M}$ gamitrinib for $24 \mathrm{~h}$. Statistical significance with respect to vehicle-treated cells: ${ }^{*} P=0.0002 ;{ }^{\circ} P=0.003 ; * * P=0.002 ;{ }^{\circ} P=0.0001$. (D) Apoptotic levels in ML1, FTC133, BHT101, CAL62, and BCPAP cells pretreated with $1 \mu \mathrm{M}$ gamitrinib for $6 \mathrm{~h}$ and subsequently exposed to $1 \mu \mathrm{M}$ paclitaxel for $24 \mathrm{~h}(0.25 \mu \mathrm{M}$ in BHT101 cells, $0.5 \mu \mathrm{M}$ in BCPAP cells). Statistical significance with respect to cells treated with paclitaxel as single agent: ${ }^{*} P=0.0003 ;{ }^{*} P=0.001{ }^{\circ} P=0.0008$; ${ }^{\circ} P=0.003$. 
A $\quad$ Control $\square$ siTRAP1

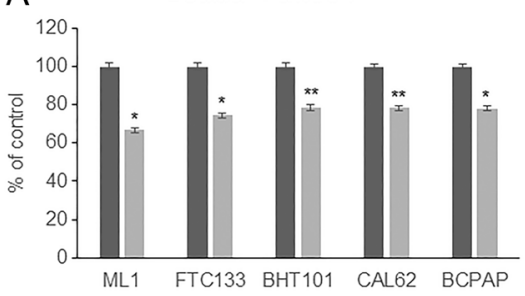

B

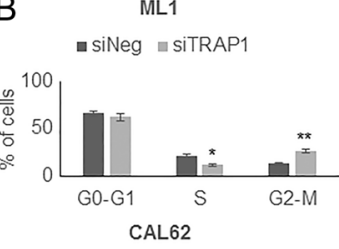

$\because$ siNeg $=$ SiTRAP1
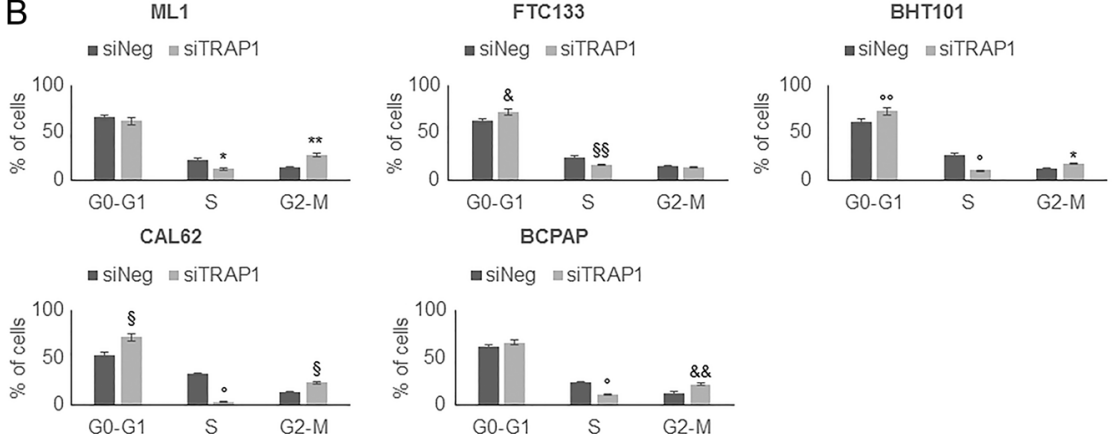

siNeg $\square$ SiTRAP1

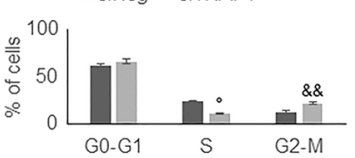

Figure 3

TRAP1 favors cell cycle progression. (A) ML1, FTC133, BHT101, CAL62 and BCPAP cells were transfected with control or TRAP1 siRNAs and counted after $72 \mathrm{~h}$. Statistical significance with respect to negative siRNA cells: ${ }^{*} P<0.0001$; $* * P=0.0001$. Insert: TRAP1 immunoblot analysis in ML1, FTC133, BHT101, CAL62 and BCPAP cells transfected with control and TRAP1 siRNAs.

(B) Cell cycle distribution in ML1, FTC133, BHT101, CAL62 and BCPAP cells transfected with control or TRAP1 siRNAs and analyzed $48 \mathrm{~h}$ after siRNA transfection. Statistical significance with respect to negative siRNA cells: ${ }^{*} P=0.001 ; * * P=0.0002$;

${ }^{\circ} P<0.0001 ;{ }^{\circ} P=0.01 ; \S P=0.002 ; \S \S P=0.0005$; $\& P=0.03 ; \& \& P=0.0008$. cell proliferation (Fig. 5A) and cell cycle progression (Fig. 5B) in our panel of TC cell lines. As observed for gamitrinib, HSP990 activity was independent from BRAF mutational status (Fig. 5). These data suggest that TRAP1 may represent a novel therapeutic target in PDTCs.

\section{Gamitrinib and HSP990 cytotoxic/cytostatic activity depends on TRAP1 inhibition}

In order to demonstrate that the observed cytotoxic/ cytostatic activities showed by gamitrinib and HSP990 depend on TRAP1 targeting, both agents were tested in
A

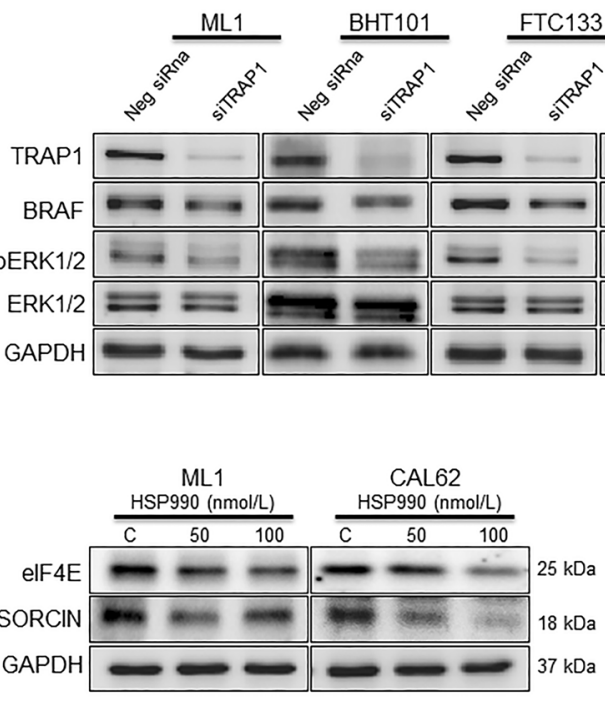

C

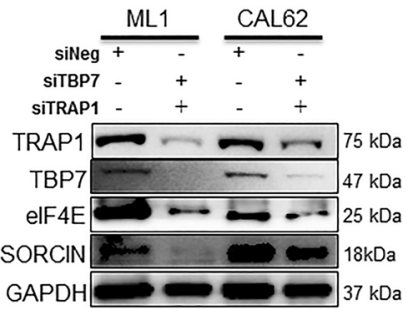

D

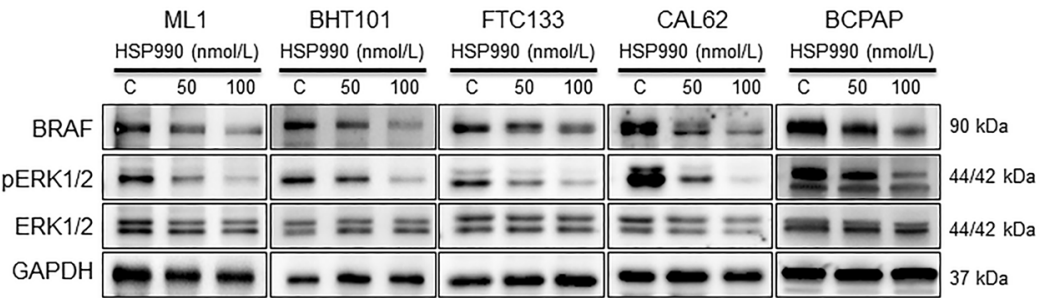

Figure 4

TRAP1 silencing/inhibition results in attenuation of ERK signaling. (A) Immunoblot analysis of BRAF, TRAP1, ERK and phosphoERK protein levels in ML1, FTC133, BHT101, CAL62 and BCPAP cells $72 \mathrm{~h}$ after TRAP1 silencing. ( $B$ and $C$ ) Immunoblot analysis of Sorcin and eIF4E protein levels in ML1 and CAL62 cells treated with 50 or $100 \mathrm{nM}$ HSP990 for 24h (B) or transfected with TRAP1 and TBP7 siRNAs (C). (D) Immunoblot analysis of BRAF, TRAP1, ERK and phosphoERK protein levels in ML1, FTC133, BHT101, CAL62 and BCPAP cells treated with 50 or $100 \mathrm{nM} \mathrm{HSP990}$ for $24 \mathrm{~h}$. 
A

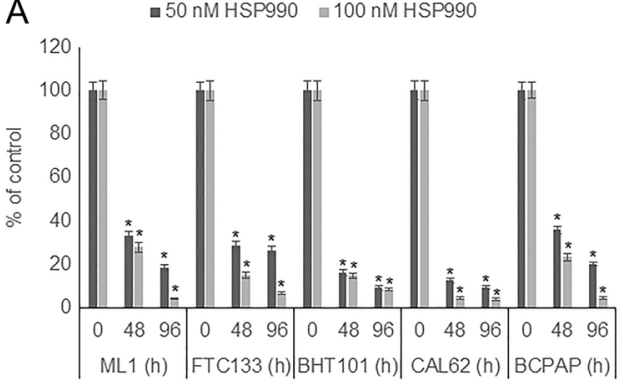

B

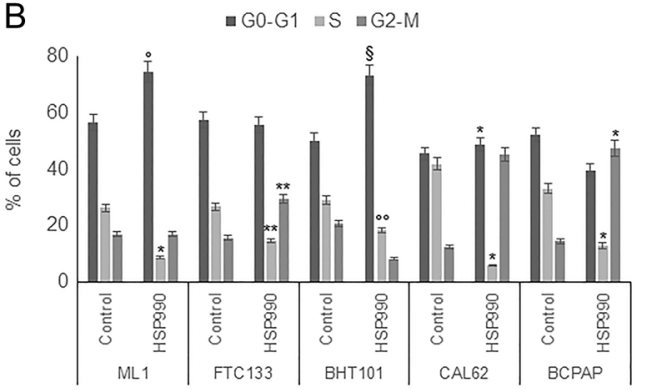

C

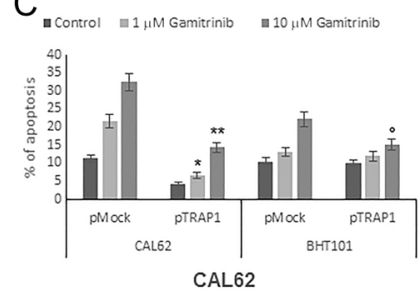

D

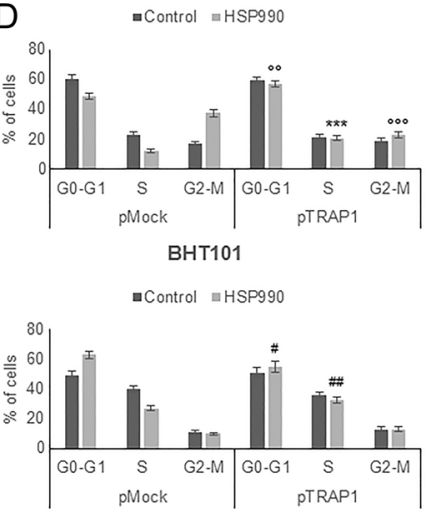

\section{Figure 5}

HSP90/TRAP1 targeting results in attenuation of cell proliferation and cell cycle progression. (A) ML1, FTC133, BHT101, CAL62 and BCPAP cells were plated in 6-well plates, treated with 50 and $100 \mathrm{nM}$ HSP990 and counted after 48 and $96 \mathrm{~h}$. Statistical significance with respect to vehicletreated cells: ${ }^{*} P 0.0001$. (B) Cell cycle distribution in ML1, FTC133, BHT101, CAL62 and BCPAP cells treated with $100 \mathrm{nM} \mathrm{HSP990}$ for $24 \mathrm{~h}$. Statistical significance with respect to vehicle-treated cells: ${ }^{*} P<0.0001 ;{ }^{* *} P=0.0001 ;{ }^{\circ} P=0.002 ;{ }^{\circ} P=0.0004$; $\S P=0.0008$. (C and D). Apoptotic levels (C) and cell cycle distribution (D) in CAL62 and BHT101 cells transfected with pMock or TRAP1 CDNA and treated with 1 or $10 \mu \mathrm{M}$ gamitrinib (C) or $100 \mathrm{nM}$ HSP990 for $24 \mathrm{~h}$. Statistical significance with respect to pMock cells: $* P=0.0002 ; * * P=0.0003$; ${ }^{* * * P} P=0.002 ;{ }^{\circ} P=0.007 ;{ }^{\circ 0} P=0.01 ;{ }^{\circ 00} P=0.001$; ${ }^{\#} P=0.03,{ }^{\# \#} P=0.02$.
CAL62 and BHT101 cells upon transfection of TRAP1 cDNA (Supplementary Fig. 2B and C). Of note, either gamitrinib or HSP990 failed to induce, respectively, apoptosis (Fig. 5C and Supplementary Fig. 2B) and inhibit cell cycle (Fig. 5D and Supplementary Fig. 2C) in a high TRAP1 background. These evidences suggest that TRAP1 targeting is relevant for the cytotoxic/cytostatic activity of both HSP90 inhibitors.

\section{Discussion}

The rationale for evaluating TRAP1 role in TC progression relies on recent evidences suggesting a key involvement of this HSP90 molecular chaperone in human carcinogenesis (Amoroso et al. 2014, Rasola et al. 2014). Indeed, TRAP1 multifaceted functions in protection from apoptosis, regulation of cell cycle, cell metabolism and protein homeostasis have been widely demonstrated in several human malignancies (Kang et al. 2007, Amoroso et al. 2012, Matassa et al. 2013, Condelli et al. 2014, Rasola et al. 2014). However, controversial data have been reported on whether TRAP1 behaves as oncogene or oncosuppressor, being its expression levels increased in the majority of human malignancies (Costantino et al. 2009, Maddalena et al. 2013, Agorreta et al. 2014, Rasola et al. 2014), but downregulated along with increased tumor grading in specific human tumors (i.e. ovarian, cervical and kidney carcinomas) (Yoshida et al. 2013, Matassa et al. 2016). This issue is relevant in human TC cells since activation of ERK signaling with parallel loss of TSHR signaling and thyroid-specific characters correlates with worsening of clinical outcome (Menezes et al. 2012, Regalbuto et al. 2012, Patel \& Shaha 2014). Interestingly, while the majority of TCs are well-differentiated tumors with excellent prognosis, a subset of TCs are characterized by a more aggressive biological and clinical behavior, unresponsiveness to radioiodine therapy and chemotherapeutics and increased metastatic dissemination (Mazzaferri \& Massoll 2002, Regalbuto et al. 2012). In this context, the validation of biomarkers for early detection of these more aggressive TCs and the development of new therapeutic strategies targeting molecular mechanisms responsible for the aggressive behavior of these thyroid malignancies are relevant objectives to improve the prognosis of these patients (Omur \& Baran 2014).

Thus, TRAP1 expression was analyzed in a large series of TCs with different grading and stages and its involvement in driving specific features of PDTC cells (i.e., increased cell proliferation and resistance to apoptosis) was studied in vitro. Remarkably, TRAP1 expression is induced along tumor (de)differentiation: (i) in microfollicular compared with macrofollicular areas of normal thyroid gland, (ii) in well-differentiated TCs compared with normal tissues, and (iii) in the transition from well-differentiated to PDTCs and in FV-PTCs, two tumor entities with worst biological and clinical phenotypes compared with well-differentiated TCs (Vivero et al. 2013, Yu et al. 2013, Patel \& Shaha 2014). Consistently, in vitro experiments suggest that TRAP1 is involved in the regulation of cell cycle progression and the apoptotic

Published by Bioscientifica Ltd. 
threshold of TC cells. In this context, it is worth noting that TRAP1 knocking down correlates with increased sensitivity to paclitaxel, reduction of S-phase fraction of cell cycle, and attenuation of ERK signaling, a pathway known to drive TC cell (de)differentiation (Nikiforov et al. 2011), thus suggesting that TRAP1 upregulation is likely responsible for TC progression. Conflicting with this evidence is the observation that TRAP1 expression is completely lost in a subset of high-grade ATCs, being conversely upregulated in the majority of these highly aggressive tumors (11/16 cases in our cohort). Although this observation cannot be easily argued, it is intriguing to speculate that a subset of ATCs may be characterized by a different metabolic and biologic phenotype (Ragazzi et al. 2014) and the loss of TRAP1 may be relevant in favoring these differences. This hypothesis is reinforced by the recent observation that TRAP1 is downregulated in selected malignancies along with increased tumor grading (Yoshida et al. 2013, Matassa et al. 2016) and that TRAP1 may enhance or inhibit oxidative phosphorylation in cancer cells based on the tumor type or the cell context (Rasola et al. 2014). This observation suggests that the loss of TRAP1 expression in specific tumor types/conditions may be functional to metabolic reprogramming of cancer cells. However, the limited number of ATCs in our series does not allow to rule out/confirm the hypothesis that these TRAP1-negative ATCs may represent a different biological entity, which represents a topic that deserves further investigation.

Relevant is the observation that TRAP1 silencing induces a profound attenuation of ERK phosphorylation. Indeed, a large subgroup of thyroid malignancies is characterized by the activation of the RAS/RAF/ERK pathway due to activating mutations/rearrangement of RET/PTC, BRAF or RAS genes, which results in more aggressive clinical phenotypes (Riesco-Eizaguirre et al. 2006, Gao et al. 2012, Omur et al. 2014, Yang et al. 2014). Furthermore, this biological evidence was translated at clinical level in the development of novel anticancer therapies targeting this signaling axis (Fallahi et al. 2015). Intriguingly, TRAP1 silencing induced the parallel downregulation of BRAF and ERK signaling in follicular $B R A F / R A S$ wild-type ML1 and FTC133 cells and BRAFV600E BHT101 and BCPAP cells, but only the downregulation of ERK phosphorylation in KRAS-mutated CAL62 cells. Indeed, while this is the first observation that TRAP1 is responsible for the modulation of ERK signaling independently from its regulatory activity on BRAF synthesis (Condelli et al. 2014), it supports the hypothesis that TRAP1 targeting may represent a strategy to inhibit ERK pathway in TCs. However, whether the lack of correlation between BRAF expression and ERK activation in low TRAP1 background is due to the RAS mutational status of CAL62 cells or to other unknown regulatory mechanisms is still an open issue.

Clinically relevant is the evidence that TRAP1 targeting results in reversion of resistance to apoptosis and inhibition of cell proliferation. Two independent agents, targeting TRAP1 in a compartmentalized manner, achieved this result. Indeed, gamitrinib, a dual HSP90/TRAP1 ATPase inhibitor, which accumulates in mitochondria favoring the opening of the mitochondrial transition pore (Kang et al. 2009), showed a single-agent cytotoxic activity and enhanced the cytotoxicity of paclitaxel. Consistently, HSP990, which is not engineered to enter mitochondria, significantly inhibited ERK signaling, cell cycle progression and cell proliferation. HSP990 is a TRAP1 inhibitor, as previously demonstrated in other tumor cell models (Menezes et al. 2012, Condelli et al. 2014) and confirmed in TC cells by the downregulation of specific TRAP1 client proteins. It is also an inhibitor of HSP90 and likely other HSP90 chaperones, as their ATPase domains are highly homologous. Indeed, this multiple inhibitory activity may account for some differences in cell cycle inhibition profiles observed between HSP990treated and TRAP1-silenced TC cells and for the dramatic difference between its cytostatic activity and the effect of TRAP1 silencing on cell proliferation. Overall, these observations suggest that HSP90 chaperones may represent valuable molecular targets to develop novel therapeutic strategies in radioiodine-refractory TCs, a subset of TCs with poor prognosis and limited therapeutic options (Xing et al. 2013). In such a context, the MEK inhibitor selumetinib showed interesting re-differentiating activity against radioiodine-refractory advanced TCs, producing clinically meaningful increases of iodine uptake and retention (Ho et al. 2013) and similar observations were reported upon treatment of BRAF-V600E-mutant PTCs with the BRAF inhibitor dabrafenib (Rothenberg et al. 2015). Intriguingly, HSP990 downregulated BRAF in TC cells and induced a significant downregulation of cell proliferation independently from $B R A F$ mutational status, thus suggesting that HSP90/TRAP1 pharmacological targeting deserves to be evaluated as a strategy to inhibit ERK signaling in radioiodine-refractory TCs. This issue is becoming relevant considering the recent development of the first mitochondria-targeted HSP90 inhibitor designed on the crystal structure of human TRAP1 (Lee et al. 2015).

Supplementary data

This is linked to the online version of the paper at http://dx.doi.org/10.1530/ ERC-16-0063.

Published by Bioscientifica Ltd. 


\section{Declaration of interest}

The authors declare that there is no conflict of interest that could be perceived as prejudicing the impartiality of the research reported.

\section{Funding}

This work was supported by Associazione Italiana per la Ricerca sul Cancro (AIRC) (Grant IG2015 Id.16738 to M L and F E), Italian Ministry of Health (Grant GR-2010-2310057 to M L) and University of Foggia (PRA Grant to $\mathrm{ML}$ ).

\section{Author contribution statement}

Study concept and design, M C, F E, M L; acquisition of data, G P, G P, A P, T N, L S, A S, G S; analysis and interpretation of data, G P, G P, P T, P B, $F E, M$ L; drafting of the manuscript, G P, G P, M L; critical revision of the manuscript, $M$ C, F E, M L; statistical analysis, O L, G S; obtained funding, $F$ E, M L; study supervision, M L.

\section{References}

Agorreta J, Hu J, Liu D, Delia D, Turley H, Ferguson DJ, Iborra F, Pajares MJ, Larrayoz M, Zudaire I, et al. 2014 TRAP1 regulates proliferation, mitochondrial function, and has prognostic significance in NSCLC. Molecular Cancer Research 12 660-669. (doi:10.1158/1541-7786.MCR-13-0481)

Alonso-Gordoa T, Diez JJ, Duran M \& Grande E 2015 Advances in thyroid cancer treatment: latest evidence and clinical potential. Therapeutic Advances in Medical Oncology 7 22-38. (doi:10.1177/1758834014551936)

Amoroso MR, Matassa DS, Laudiero G, Egorova AV, Polishchuk RS, Maddalena F, Piscazzi A, Paladino S, Sarnataro D, Garbi C, et al. 2012 TRAP1 and the proteasome regulatory particle TBP7/Rpt3 interact in the endoplasmic reticulum and control cellular ubiquitination of specific mitochondrial proteins. Cell Death and Differentiation 19 592-604. (doi:10.1038/cdd.2011.128)

Amoroso MR, Matassa DS, Sisinni L, Lettini G, Landriscina M \& Esposito F 2014 TRAP1 revisited: novel localizations and functions of a 'next-generation' biomarker (review). International Journal of Oncology 45 969-977. (doi:10.3892/ijo.2014.2530)

Aust S, Bachmayr-Heyda A, Pateisky P, Tong D, Darb-Esfahani S, Denkert C, Chekerov R, Sehouli J, Mahner S, Van Gorp T, et al. 2012 Role of TRAP1 and estrogen receptor alpha in patients with ovarian cancer - a study of the OVCAD consortium. Molecular Cancer 1169. (doi:10.1186/1476-4598-11-69)

Condelli V, Piscazzi A, Sisinni L, Matassa DS, Maddalena F, Lettini G, Simeon V, Palladino G, Amoroso MR, Trino S, et al. 2014 TRAP1 is involved in BRAF regulation and downstream attenuation of ERK phosphorylation and cell cycle progression: a novel target for BRAF-mutated colorectal tumors. Cancer Research 74 6693-6704. (doi:10.1158/0008-5472.CAN-14-1331)

Condelli V, Maddalena F, Sisinni L, Lettini G, Matassa DS, Piscazzi A, Palladino G, Amoroso MR, Esposito F \& Landriscina M 2015 Targeting TRAP1 as a downstream effector of BRAF cytoprotective pathway: a novel strategy for human BRAF-driven colorectal carcinoma. Oncotarget 6 22298-22309. (doi:10.18632/oncotarget)

Costantino E, Maddalena F, Calise S, Piscazzi A, Tirino V, Fersini A, Ambrosi A, Neri V, Esposito F \& Landriscina M 2009 TRAP1, a novel mitochondrial chaperone responsible for multi-drug resistance and protection from apoptotis in human colorectal carcinoma cells. Cancer Letters 279 39-46. (doi:10.1016/j. canlet.2009.01.018)
Fallahi P, Mazzi V, Vita R, Ferrari SM, Materazzi G, Galleri D, Benvenga S, Miccoli P \& Antonelli A 2015 New therapies for dedifferentiated papillary thyroid cancer. International Journal of Molecular Sciences 16 6153-6182. (doi:10.3390/ijms16036153)

Gao WL, Wie LL, Chao YG, Wie L \& Song TL 2012 Prognostic prediction of BRAF(V600E) and its relationship with sodium iodide symporter in classic variant of papillary thyroid carcinomas. Clinical Laboratory 58 919-926. (doi:10.7754/clin.lab.2012.111217)

Han JJ, Baek SK, Lee JJ, Kim GY, Kim SY \& Lee SH 2014 Combination of TRAP1 and ERCC1 expression predicts clinical outcomes in metastatic colorectal cancer treated with oxaliplatin/5-fluorouracil. Cancer Research and Treatment 46 55-64. (doi:10.4143/ crt.2014.46.1.55)

Haugen BR 2004 Redifferentiation therapy in advanced thyroid cancer. Current Drug Targets: Immune, Endocrine \& Metabolic Disorders 4 175-180. (doi:10.2174/1568008043339811)

Ho AL, Grewal RK, Leboeuf R, Sherman EJ, Pfister DG, Deandreis D, Pentlow KS, Zanzonico PB, Haque S, Gavane S, et al. 2013 Selumetinib-enhanced radioiodine uptake in advanced thyroid cancer. New England Journal of Medicine 368 623-632. (doi:10.1056/ NEJMoa1209288)

Kang BH, Plescia J, Dohi T, Rosa J, Doxsey SJ \& Altieri DC 2007 Regulation of tumor cell mitochondrial homeostasis by an organellespecific Hsp90 chaperone network. Cell 131 257-270. (doi:10.1016/ j.cell.2007.08.028)

Kang BH, Plescia J, Song HY, Meli M, Colombo G, Beebe K, Scroggins B, Neckers L \& Altieri DC 2009 Combinatorial drug design targeting multiple cancer signaling networks controlled by mitochondrial Hsp90. Journal of Clinical Investigation 119 454-464. (doi:10.1172/ JCI37613)

Kogai T \& Brent GA 2012 The sodium iodide symporter (NIS): regulation and approaches to targeting for cancer therapeutics. Pharmacology \& Therapeutics 135 355-370. (doi:10.1016/j.pharmthera.2012.06.007)

Landriscina M, Laudiero G, Maddalena F, Amoroso MR, Piscazzi A, Cozzolino F, Monti M, Garbi C, Fersini A, Pucci P, et al. 2010 Mitochondrial chaperone Trap1 and the calcium binding protein Sorcin interact and protect cells against apoptosis induced by antiblastic agents. Cancer Research 70 6577-6586. (doi:10.1158/ 0008-5472.CAN-10-1256)

Leav I, Plescia J, Goel HL, Li J, Jiang Z, Cohen RJ, Languino LR \& Altieri DC 2010 Cytoprotective mitochondrial chaperone TRAP-1 as a novel molecular target in localized and metastatic prostate cancer. American Journal of Pathology 176 393-401. (doi:10.2353/ ajpath.2010.090521)

Lee C, Park HK, Jeong H, Lim J, Lee AJ, Cheon KY, Kim CS, Thomas AP, Bae B, Kim ND, et al. 2015 Development of a mitochondria-targeted Hsp90 inhibitor based on the crystal structures of human TRAP1. Journal of the American Chemical Society 137 4358-4367. (doi:10.1021/ja511893n)

Maddalena F, Laudiero G, Piscazzi A, Secondo A, Scorziello A, Lombardi V, Matassa DS, Fersini A, Neri V, Esposito F \& Landriscina M 2011 Sorcin induces a drug-resistant phenotype in human colorectal cancer by modulating $\mathrm{Ca}(2+)$ homeostasis. Cancer Research 71 7659-7669. (doi:10.1158/0008-5472.CAN-11-2172)

Maddalena F, Sisinni L, Lettini G, Condelli V, Matassa DS, Piscazzi A, Amoroso MR, La Torre G, Esposito F \& Landriscina M 2013 Resistance to paclitxel in breast carcinoma cells requires a quality control of mitochondrial antiapoptotic proteins by TRAP1. Molecular Oncology 7 895-906. (doi:10.1016/j.molonc.2013.04.009)

Matassa DS, Amoroso MR, Agliarulo I, Maddalena F, Sisinni L, Paladino S, Romano S, Romano MF, Sagar V, Loreni F, et al. 2013 Translational control in the stress adaptive response of cancer cells: a novel role for the heat shock protein TRAP1. Cell Death and Disease 4 e851. (doi:10.1038/cddis.2013.379)

Matassa DS, Amoroso MR, Lu H, Avolio R, Arzeni D, Procaccini C, Faicchia D, Maddalena F, Simeon V, Agliarulo I, et al. 2016 
Oxidative metabolism drives inflammation-induced platinum resistance in human ovarian cancer. Cell Death and Differentiation [in press]. (doi:10.1038/cdd.2016.39)

Mazzaferri EL \& Massoll N 2002 Management of papillary and follicular (differentiated) thyroid cancer: new paradigms using recombinant human thyrotropin. Endocrine-Related Cancer 9 227-247. (doi:10.1677/erc.0.0090227)

Menezes DL, Taverna P, Jensen MR, Abrams T, Stuart D, Yu GK, Duhl D, Machajewski T, Sellers WR, Pryer NK, et al. 2012 The novel oral Hsp90 inhibitor NVP-HSP990 exhibits potent and broad-spectrum antitumor activities in vitro and in vivo. Molecular Cancer Therapeutics 11 730-739. (doi:10.1158/15357163.MCT-11-0667)

Montesano Gesualdi N, Chirico G, Pirozzi G, Costantino E, Landriscina M \& Esposito F 2007 Tumor necrosis factor-associated protein 1 (TRAP-1) protects cells from oxidative stress and apoptosis. Stress 10 342-350. (doi:10.1080/10253890701314863)

Mu D, Huang R, Li S, Ma X, Lou C \& Kuang A 2012 Combining transfer of TTF- 1 and Pax- 8 gene: a potential strategy to promote radioiodine therapy of thyroid carcinoma. Cancer Gene Therapy 19 402-411. (doi:10.1038/cgt.2012.13)

Nikiforov YE \& Nikiforova MN 2011 Molecular genetics and diagnosis of thyroid cancer. Nature Reviews Endocrinology 7 569-580. (doi:10.1038/ nrendo.2011.142)

Omur O \& Baran Y 2014 An update on molecular biology of thyroid cancers. Critical Reviews in Oncology/Hematology 90 233-252. (doi:10.1016/j.critrevonc.2013.12.007)

Pannone G, Santoro A, Pasquali D, Zamparese R, Mattoni M, Russo G, Landriscina M, Piscazzi A, Toti P, Cignarelli M, et al. 2014 The role of survivin in thyroid tumors: differences of expression in well-differentiated, non-well-differentiated, and anaplastic thyroid cancers. Thyroid 24 511-519. (doi:10.1089/thy.2013.0196)

Patel KN \& Shaha AR 2014 Poorly differentiated thyroid cancer. Current Opinion in Otolaryngology \& Head and Neck Surgery 22 121-126. (doi:10.1097/MOO.0000000000000037)

Piscazzi A, Costantino E, Maddalena F, Natalicchio MI, Gerardi AM, Antonetti R, Cignarelli M \& Landriscina M 2012 Activation of the RAS/RAF/ERK signaling pathway contributes to resistance to sunitinib in thyroid carcinoma cell lines. Journal of Clinical Endocrinology and Metabolism 97 E898-E906. (doi:10.1210/ jc.2011-3269)

Ragazzi M, Ciarrocchi A, Sancisi V, Gandolfi G, Bisagni A \& Piana S 2014 Update on anaplastic thyroid carcinoma: morphological, molecular, and genetic features of the most aggressive thyroid cancer. International Journal of Endocrinology 2014790834. (doi:10.1155/2014/790834)
Rasola A, Neckers L \& Picard D 2014 Mitochondrial oxidative phosphorylation TRAP(1)ped in tumor cells. Trends in Cell Biology 24 455-463. (doi:10.1016/j.tcb.2014.03.005)

Regalbuto C, Frasca F, Pellegriti G, Malandrino P, Marturano I, Di Carlo I \& Pezzino V 2012 Update on thyroid cancer treatment. Future Oncology 8 1331-1348. (doi:10.2217/fon.12.123)

Riesco-Eizaguirre G, Gutierrez-Martinez P, Garcia-Cabezas MA, Nistal M \& Santisteban P 2006 The oncogene BRAF V600E is associated with a high risk of recurrence and less differentiated papillary thyroid carcinoma due to the impairment of $\mathrm{Na}+/ \mathrm{I}$ - targeting to the membrane. Endocrine-Related Cancer 13 257-269. (doi:10.1677/ erc.1.01119)

Rothenberg SM, McFadden DG, Palmer EL, Daniels GH \& Wirth LJ 2015 Redifferentiation of iodine-refractory BRAF V600E-mutant metastatic papillary thyroid cancer with dabrafenib. Clinical Cancer Research 21 1028-1035. (doi:10.1158/1078-0432.CCR-14-2915)

Smith NE, Illei PB, Allaf M, Gonzalez N, Morris K, Hicks J, Demarzo A, Reuter VE, Amin MB, Epstein JI, et al. 2014 t $(6 ; 11)$ renal cell carcinoma (RCC): expanded immunohistochemical profile emphasizing novel RCC markers and report of 10 new genetically confirmed cases. American Journal of Surgical Pathology 38 604-614. (doi:10.1097/pas.0000000000000203)

Vivero M, Kraft S \& Barletta JA 2013 Risk stratification of follicular variant of papillary thyroid carcinoma. Thyroid 23 273-279. (doi:10.1089/thy.2012.0369)

Wada N, Nakayama H, Suganuma N, Masudo Y, Rino Y, Masuda M \& Imada T 2007 Prognostic value of the sixth edition AJCC/UICC TNM classification for differentiated thyroid carcinoma with extrathyroid extension. Journal of Clinical Endocrinology and Metabolism 92 215-218. (doi:10.1210/jc.2006-1443)

Xing M, Haugen BR \& Schlumberger M 2013 Progress in molecularbased management of differentiated thyroid cancer. Lancet $\mathbf{3 8 1}$ 1058-1069. (doi:10.1016/S0140-6736(13)60109-9)

Yang K, Wang H, Liang Z, Liang J, Li F \& Lin Y 2014 BRAFV600E mutation associated with non-radioiodine-avid status in distant metastatic papillary thyroid carcinoma. Clinical Nuclear Medicine 39 675-679. (doi:10.1097/RLU.0000000000000498)

Yoshida S, Tsutsumi S, Muhlebach G, Sourbier C, Lee MJ, Lee S, Vartholomaiou E, Tatokoro M, Beebe K, Miyajima N, et al. 2013 Molecular chaperone TRAP1 regulates a metabolic switch between mitochondrial respiration and aerobic glycolysis. PNAS 110 E1604-E1612. (doi:10.1073/pnas.1220659110)

Yu XM, Schneider DF, Leverson G, Chen H \& Sippel RS 2013 Follicular variant of papillary thyroid carcinoma is a unique clinical entity: a population-based study of 10,740 cases. Thyroid 23 1263-1268. (doi:10.1089/thy.2012.0453)

Received in final form 3 July 2016

Accepted 14 July 2016

Accepted Preprint published online 15 July 2016
(C) 2016 Society for Endocrinology Printed in Great Britain
Published by Bioscientifica Ltd. 